\title{
The preliminary study of diagnosis techniques for the building defects with damp by infrared thermography
}

\author{
Longyu Wan ${ }^{1, a}$ and Zukang Lei $\mathrm{i}^{2, \mathrm{~b}}$ * \\ ${ }^{1}$ School of Architecture and Urban Planning, Huazhong University of Science and Technology 430074, \\ Wuhan, Hubei, China. \\ adora.one@qq.com, bukanglei@126.com
}

\begin{abstract}
Keywords: Infrared Thermography, Building Damp, Building Defect, Field Diagnosis Technique, Building Pathology

Abstract. Since a large amount of building defects arise from inside and are invisible at the beginning, the most common methodologies to assess defects are not able to predict a new defect or extension of an existed one. On this study, infrared thermal imaging technology and traditional methods of building diagnosis were used in the investigation of a external wall on the west side of an old building. This study intends to find dampness in thermal image which is invisible in visible light image and the relationships between variation of surface temperature and building defects. The results of this study indicate that infrared thermography provides information for the study of diagnosis techniques of the building defects.
\end{abstract}

\section{Introduction}

In China, building diagnosis is not widely applied, part of the reason is that most people don't take the "problem" seriously and are not aware of the damage of housing defects. Lack of a reasonable operating method is also a contributing factor to it. Inspecting building defects using a thermal imaging camera is a modern non-destructive measuring method of monitoring and diagnosing the condition of buildings. Normally, building renovators identify with their eyes. However, a thermal imaging camera can see what is invisible such as rising damp, which provides more evidence to judge building condition.

\section{Traditional methods of diagnosing building defects}

The object of this study is a brick wall in Huazhong University of Science and Technology, Wuhan, Hubei, China. It belongs to the teachers' estate built in 1960s and has been suffering from serious housing defects over these years. As the wall is surrounded by trees, there is not enough lighting, then mould and moisture grows. (Fig. 1)

The traditional way of diagnosing defects in China relies on visible features of various defects and acknowledgments of inspector. Using the traditional strategy of building diagnostic the following defects were sorted:

Defects on external wall surface. Plaster and render of a wall faces attack both from outside and inside. If deterioration shows on wall surface, it means source of a defect has stayed for a period of time and may lead to a more severe one.

Detachment of render. The detached render occupies nearly two thirds of the whole wall from bottom, showing the masonry background. The defect is more severe on the left side, it is probably caused by pipe leaks and frost attack, and holes for new drainpipe on the left side of the wall. (Fig. 2)

Peeling and flaking. On plaster peeling and flaking can be found allover except the area under eaves. Commonly, it's caused by the cross-effect of long-term dampness and evaporation, which makes plaster fragile. Surface around drainpipe is also facing peeling caused by pipe leakage. (Fig. 3)

Efflorescence. Efflorescence is caused by soluble salts being brought to the surface as water in the wall dries out. In this case it produces a white staining on all over render of the wall. The staining remains at the left and right corner of the wall and around drainpipe in the middle of the wall. Moisture in environment usually starts from verge because render there is vulnerable and it lacks of circulation of air in the room corner, which 
means water comes from both ground and environment. Efflorescence around drainpipe is forming peeling and flaking as said above. Form of the staining is like an outline of splash. As moisture is sufficient in this area due to pipe leakage, crystallization of salts can cause spalling of plaster, which means water comes from environment. (Fig. 4)

Mould growth. On plaster mould appears near pipe in the middle of wall. The closer to pipe, the more the mould. Defect changes itself mildly from detachment, mould to efflorescence as it leaves the pipe. If mould stays growing, it may lead to a more severe damage. (Fig. 5)

Iron staining. It occurs only on surface below the two pipes, as water rises and moves, rust remains and piles up.

Drainpipe break. Drainpipe in the middle was broken and is not in use, but the defects remain. Despite of the hole in upper part, pipe at the bottom has already fractured. The original material on the bottom of right pipe was replaced by a plastic one, and is already showing deterioration. It is probably because the end of pipe is too close to open drain. As a result, fallen leaves and mud have blocked the drain.

Defects on masonry background. In this case about two thirds of the masonry background is exposed. Defects on masonry background are different from those on plaster and render because of material and moisture source. Defects on different layers should be considered separately.

Efflorescence. On masonry efflorescence is all over and that around drainpipe and under protection of window ledges is causing concern. As moisture rises over plinth-wall and salts solute, the staining remains about one meter above ground. (Fig. 4)

Lime run-off. As salts crystallize, they can cause decay in mortar joints. Water can dissolve calcium hydroxide (free lime) in the cement of mortar joints which is then react with carbon dioxide in the air, producing calcium carbonate. In this case run-off appears by the edge of two windows on the right, which means void in mortar beds has formed weep holes. (Fig. 6)

Iron staining. As is said above, iron pipes get rusty and staining attaches to wall surface, including plaster layer and exposed masonry background.

Mould growth. Area of mould growth on masonry is around end of pipe or leaking joints because of high moisture content of wall. (Fig. 5)

Plant growth. Plant growth on joints of masonry and plinth-wall is the result of rising damp and penetrating damp. Moisture from below ground and leaking pipes provides water, without cover of render, soil and debris blocked void in mortar beds, these two factors contribute to plant growth over the years. (Fig. 7)

Missing bricks. There are six places of missing bricks due to human factor. Two of them has been poorly replaced, the other are prepared for new drainage system from interior.

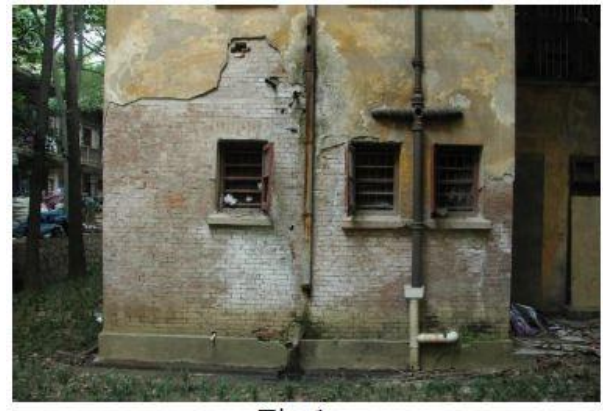

Fig.1.

Visible light image.

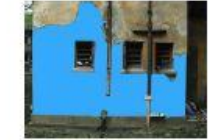

Fig. 2 .

Detachment of render.

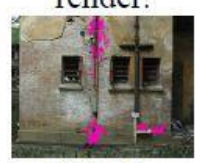

Fig. 5. Mould growth.

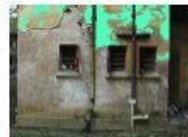

Fig. 3.

Peeling and flaking.

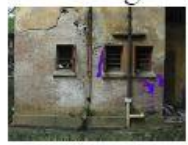

Fig. 6.

Lime run-off.

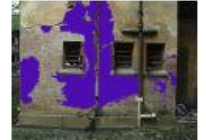

Fig. 4.

Efflorescence.

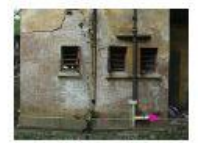

Fig. 7.

Plant growth.

\section{Thermal image analysis}

An example of analyzing wall surface temperature gradient from thermal image containing various defects. A line measurement in thermogram which represents the most typical defects on surface was exported to csv file (Fig. 8). As seen from Fig. 9 three kinds of defects can be figured out (but there is some overlap on the curve) according to their gradients: 
Efflorescence. Its temperature ranges from $23.9{ }^{\circ} \mathrm{C}$ to $25.4{ }^{\circ} \mathrm{C}$, and its gradient, ratio of surface temperature change and number of measure points is 0.028 .

Rising damp. Its temperature ranges from $20.0{ }^{\circ} \mathrm{C}$ to $24.5{ }^{\circ} \mathrm{C}$, and its gradient is 0.15 .

Mould growth. Its temperature ranges from $20.0{ }^{\circ} \mathrm{C}$ to $23.0{ }^{\circ} \mathrm{C}$, its gradient is 0.3 .

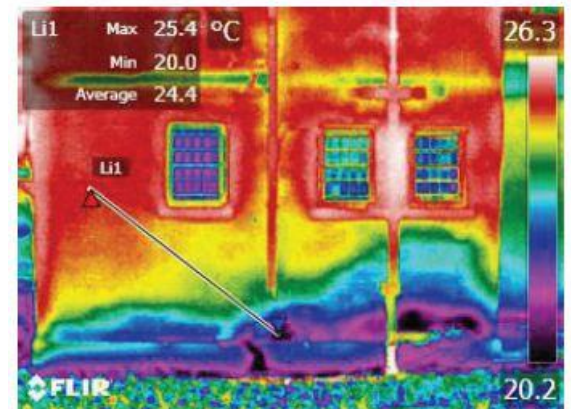

Fig. 8.

A line measurement thermal image.

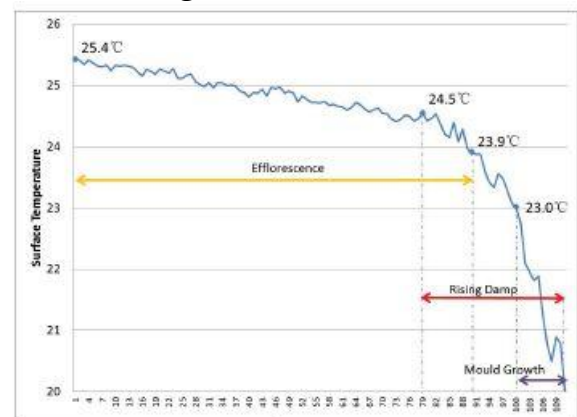

Fig. 9.

Surface temperature range of various defects on wall. The properties of other defects can be obtained in the same way. There are totally eight defects, including what is invisible (Table 1). The outlines of various defects detected with infrared thermography are shown in Fig. 10.

Table 1. Surface temperature range of various defects

\begin{tabular}{|l|l|l|}
\hline Defect & Surface temperature & Basis of determination \\
\hline Pipe deterioration & $22.8 \sim 28.7^{\circ} \mathrm{C}$ & $\begin{array}{l}\text { Its surface temperature differs a lot from the same } \\
\text { material. }\end{array}$ \\
\hline Mould growth & $19.6 \sim 23.9^{\circ} \mathrm{C}$ & $\begin{array}{l}\text { Fungi that causes decay, it's usually the darkest areas } \\
\text { in thermal image. }\end{array}$ \\
\hline Rising damp & $\begin{array}{l}\text { A large pattern of dark colors and its temperature } \\
\text { appears to get lower as it is closer to the ground. }\end{array}$ \\
\hline Pipe leakage & $\begin{array}{l}\text { The pipe is broken or it's dark around pipe in thermal } \\
\text { image. }\end{array}$ \\
\hline Efflorescence & $23.9 \sim 24.6^{\circ} \mathrm{C}$ & White staining on wall. \\
\hline Peeling and flaking & $23.9 \sim 25.1^{\circ} \mathrm{C}$ & $\begin{array}{l}\text { A small flat piece or small part of render broken or } \\
\text { detached from wall surface. }\end{array}$ \\
\hline Detachment of render & $25.1 \sim 26.7^{\circ} \mathrm{C}$ & Masonry background exposes. \\
\hline Missing bricks & $21.1 \sim 25.6^{\circ} \mathrm{C}$ & The bricks are missing or replaced. \\
\hline
\end{tabular}

\section{Defect diagnosed by themal patterns.}

Rising damp. Visible light image shows that various damp defects happen on the bottom of wall, which indicates dampness. On thermal image, contours of surface temperature at lower part are dense compares to that at upper part. This change start from about one meter high above the ground, change rate of temperature become higher as closer to the ground, so is the moisture content. This pattern is typical of rising damp. Dampness rises higher on the right, a possible explanation is that pipe in the middle is discarded but the right one is still in use, although they are all broken. (Fig. 11)

Efflorescence. Efflorescence caused by rising damp is happening below 1.5 meter above ground in thermal image, its surface temperature ranges from 23.9 to $25^{\circ} \mathrm{C}$. The vertical pattern is the result of pipe leakage, its surface temperature ranges from 24.8 to $25.6^{\circ} \mathrm{C}$. (Fig. 12)

Mould growth. The coolest(black) areas in thermal image are mould on exposed masonry. The surrounding thermal patterns are radial and are exactly the same as it's seen in visible photo.

Pattern of mould on render in thermal image is similar to visible pattern but is smaller. It's mainly because moisture content reduced and where mould grows has become dry. (Fig. 13)

Plant growth. In thermal image, plant grows in the coolest area but its temperature is $3^{\circ} \mathrm{C}$ higher than the surrounding areas. It's confusing because thermal patterns are influenced by leaves of the plant. (Fig. 13) 
Pipe deterioration. A white (highest temperature) area in thermogram is causing concern, its temperature differs a lot from the those of the same material, properties of the material changes as it is decayed by moisture or creatures. (Fig. 14)

Missing bricks. There are four holes of missing bricks, its temperature is much lower than the surrounding area. (Fig. 15)

Wall decoration. Temperature of area between windows on the right rise sharply. It's strange because there is no deterioration in this area, a possible explanation is that decorative rendering produces void and in thermal image it looks like hollowing. (Fig. 16)

Upper floor slab. Upper floor slab is obvious in thermal image due to detachment of render. The left side of slab is exposed and its surface temperature is much higher than the surrounding areas. The right side of slab, however, is covered by render but it still shows some warm color, it's probably because poor insulation of render. (Fig. 17)

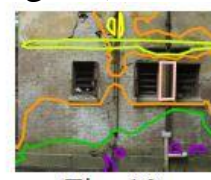

Fig. 10

Outlines of various defects.

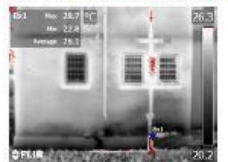

Fig. 14.

Pipe deterioration.

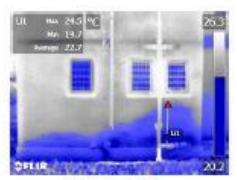

Fig. 11. Rising damp.

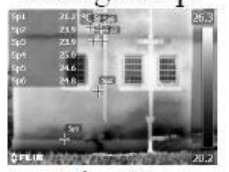

Fig. 15. Missing bricks.

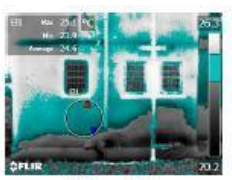

Fig. 12 . Efflorescence.

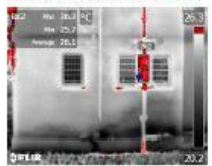

Fig. 16 Wall decoration.

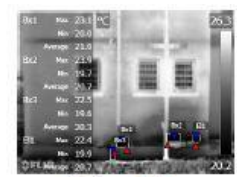

Fig. 13. Plant growth

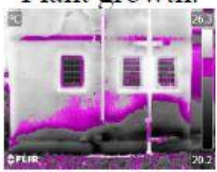

Fig. 17. Upper floor slab.

\section{Conclusions}

Infrared thermography is a new method of building diagnosis to improve the traditional way and to judge deterioration rate of a defect. Sometimes the area of a defect is not the same in visible image and thermal image, which proves that an "invisible" defect exists. This study analyses thermal patterns in two ways: one is to measure temperature of wall surface and evaluate which defect is corresponding; the other is to calculate wall surface temperature gradient and find which defect it matches to. From the diagnostic study presented in this paper, it is deduced that infrared thermography is a qualitative form of defect assessment and prediction. Thus, infrared thermography ought to be considered as a supplementary approach in building diagnosis.

\section{Acknowledgments}

This work was supported by 'the Fundamental Research Funds for the Central Universities', HUST: 2015TS066"and supported by the Scientific Research Foundation ofHUST 2014 (Grant No.0124220023).

\section{References}

[1] Raluca Pleșu, Gabriel Teodoriu and George Țăranu, Infrared thermography applications for building investigation, Bul. Inst. Polit. Iași, t. LVIII (LXII), f. 1, (2012) 157-168.

[2] N.P. Avdelidis, A. Moropoulou, P. Theoulakis, Detection of water deposits and movement in porous materials by infrared imaging, Infrared Physics \& Technology 44 (2003) 183-190.

[3] Lew Harriman, A visual moisture-detection method: Using infrared imaging to locate moisture in buildings, HPAC Engineering (2004) 23-29.

Reference to a book: 
[4] Duncan Marshall, Derek Worthing, Roger Health, Nigel Dann, Understanding Housing Defects, fourth ed., Routledge, New York, 2014. 